\title{
Veränderungen der medizinischen Wahrnehmung und des Umgangs mit sexualisierter Gewalt gegen Kinder
}

Jörg M. Fegert

Die klinische Medizin ist gekennzeichnet durch die Abfolge „sehen, kombinieren und verstehen“. Als Gegenmodell zur klassischen, diagnostischen Methode gilt nicht ohne Grund die „sprechende Medizin“. Sie blickt nicht primär auf den Patienten, sondern spricht mit ihm und ringt mit ihm um ein Problemverständnis. Im Folgenden will ich versuchen, Facetten und Wandlungen des fachlichen Diskurses in Bezug auf Opfer sexualisierter Gewalt darzustellen.

\section{Das Ausmaß sexuellen Missbrauchs}

Die Verbreitung von medizinisch relevanten Ereignissen in der Bevölkerung oder Subpopulationen ist Gegenstand der Epidemiologie. Durkheims „Le suicide" und seine methodische Schrift "Les règles de la méthode sociologique“ standen am Anfang der psychiatrischen Epidemiologie. Freuds Distanzierung von der Wahrnehmung der traumatischen Genese der von ihm beobachteten psychischen Phänomene war begründet mit dem Argument, wenn diese Narrative der Patienten stimmten und keine Phantasien wären, müssten solche Übergriffe sehr häufig sein. Seit den 1970er Jahren hat man begonnen, die tatsächliche Dimension sexualisierter Gewalt an Kindern zu ermitteln.

Erste epidemiologische Studien wurden Ende der 1970er Jahre in den USA veröffentlicht (Finkelhor 1979). Für Deutschland legte der Psychologe Michael Baurmann, damals wissenschaftlicher Direktor beim Bundeskriminalamt, im Jahr 1983 eine Dunkelfeldschätzung auf der Basis der Hellfelddaten der polizeilichen Kriminalstatistik vor, und die Sozialwissenschaftler Tilman Elliger und Kerstin Schötensack führten 1991 eine nicht repräsentative Studierendenbefragung durch.

Eine erste retrospektive Repräsentativbefragung von 1661 Frauen und 1580 Männern wurde 1997 von Peter Wetzels von der Kriminologischen Forschungsstelle Niedersachsen veröffentlicht. Bei einer breiten Definition sexualisierter Gewalt gegen Kinder gaben 18,1 \% der befragten Frauen und 7,3 \% der Männer an, solche Gewalt erfahren zu haben. ÜberTaten mit Körperkontakt berichteten 
9,6 \% der Frauen und 3,2 \% der Männer. Unsere eigenen Untersuchungen mit dem Instrument des "Childhood Trauma Questionnaire“, welches drei Definitionsbereiche unterscheidet, ergaben für die bundesrepublikanische Bevölkerung, dass 13,9 \% der Kinder im Laufe ihrer Kindheit bis zur Volljährigkeit Opfer von sexualisierter Gewalt (in weiterem Sinn) geworden sind; legt man eine engere Definition zugrunde, sind es immerhin noch 7,6 \% der Kinder und $2,3 \%$ erlitten massive fortgesetzte Taten mit Penetration. ${ }^{1}$

Im Vergleich zum Hellfeld, d.h. den Fällen, die aktenkundig werden, zeigen Dunkelfelduntersuchungen eine riesige Dimension. In ihrem Bericht zur europäischen Region spricht die Weltgesundheitsorganisation (WHO) davon, dass $90 \%$ der Taten in den eigentlich dafür zuständigen Institutionen nicht erkannt werden. Aufgrund von publizierten Metaanalysen zur Prävalenz (vgl. Stoltenborgh/Bakermans-Kranenburg/van IJzendoorn 2013) rechnet die WHO derzeit mit 18 Millionen Kindern, welche in Europa von sexuellem Missbrauch betroffen sind. Für das Gesundheitswesen betont die WHO, dass Investitionen in Kinderschutz „best buys for money“ sind, da "capacity building“ im Kinderschutz zu massiven Gewinnen an "quality of life years" führen könne.

Die Vereinten Nationen haben im Rahmen der „Roadmap für ein menschenwürdiges Leben“ ("The Road to Dignity by 2030“) Nachhaltigkeitsziele formuliert. Eines davon ist das Ziel, Misshandlung und Ausbeutung von Kindern, Kinderhandel und alle Formen von Gewalt und Folter an Kindern zu beenden. Zugleich wurden Indikatoren formuliert, um ein Monitoring der Entwicklung in diesem Bereich in allen Staaten der Welt zu ermöglichen. Der Indikator 16.2.3 beschreibt den Anteil an jungen Frauen und Männern zwischen 18 und 29 Jahren, die sexuelle Gewalt vor dem 18. Lebensjahr erfahren haben.

Ziel müsste es also sein, auch in Deutschland regelmäßig und systematisch die jüngste Erwachsenengeneration nach dem Erleben sexualisierter Gewalt im Kindes- und Jugendalter zu befragen, um veränderungssensible epidemiologische Daten zur Prävalenz der Thematik zu erhalten. Nach unseren Untersuchungen liegt derzeit die Häufigkeit bei einer breiteren Definition bei $10 \%$ in dieser Altersgruppe (Witt u.a. 2020). Äußere Effekte wie öffentliche Debatten oder die „MeToo"-Bewegung können allerdings zu deutlichen Veränderungen in der Mitteilungsbereitschaft führen. Dies wiederum kann den Anteil der Angaben zu sexualisierter Gewalt in Dunkelfeldstudien erhöhen. Auch diese erfassen ja nicht das wahre Ausmaß sexualisierter Gewalt gegen Kinder, sondern nur die gegenüber den Fragenden angegebenen Häufigkeiten.

1 Weitere Materialien zur Einordnung der Auftretenshäufigkeiten, Inzidenz und Prävalenz finden sich in Expertise Jud u.a. 2016 und Jud/Fegert/Finkelhor 2016. 
Prävalenzstudien zu sexuellem Missbrauch wurden auch in Institutionen durchgeführt. Eine entsprechende Repräsentativbefragung mit 2516 Teilnehmenden wurde im Mai bis Juli 2018 in Deutschland durchgeführt (Witt u.a. 2019a). Am häufigsten fanden sich Angaben zu sexuellem Missbrauch im schulischen Setting (1,4 \% der Gesamtbevölkerung) gefolgt von Übergriffen durch Sporttrainer (knapp o,3 \%) und Priestern in katholischen Einrichtungen (o,16\%). Ähnliche Häufigkeiten fanden sich für die evangelische Kirche. Quasi gleichzeitig berichtete die MHG-Studie (Dreßing u.a. 2018) über das zugänglich gemachte aktenkundige Hellfeld im Raum der katholischen Kirche. In dieser Studie wurde über 3677 Betroffene durch 1670 Täter berichtet. 4,4 \% aller Kleriker in den Jahren 1946 bis 2014 waren also aktenkundig übergriffig geworden.

Nimmt man eine Dunkelfeldschätzung auf der Basis der von uns erhobenen Zahlen in einer repräsentativen Stichprobe für die deutsche Bevölkerung vor, dann liegt mit sehr hoher Sicherheit die tatsächliche Häufigkeit zwischen mindestens 28 ooo und höchstens 280 ooo Betroffenen durch Priester in katholischen Institutionen. Das Dunkelfeld ist mit sehr hoher Wahrscheinlichkeit mindestens acht und bis zu achtzig Mal so groß wie das administrative Hellfeld der in der MHG-Studie untersuchten Kirchenakten.

Die spezielle Gefährdungssituation für Kinder und Jugendliche im Raum der katholischen Kirche wird erst deutlich, wenn man den Anteil der Priester an der männlichen Gesamtbevölkerung (14 847 entsprechend o, 035 \% im Jahr 2011) mit dem Anteil der Priester vergleicht, die in unserer Dunkelfelduntersuchung als Täter benannt wurden. Da $8 \%$ aller schweren Taten des sexuellen Missbrauchs mit vaginaler bzw. analer Penetration von Priestern begangen wurden, zeigt sich, dass von dieser Personengruppe eine signifikant höhere Gefährdung ausgeht als vom „gemeinen Durchschnittsmann“. Charakteristisch ist für solche Täter, dass sie häufig mehrere Opfer haben. Ähnlich wie in der MHG-Studie wird auch im Rahmen des durch den Film „Spotlight“ bekannten Skandals in der Diözese Boston 250 Priestern vorgeworfen, über einen Zeitraum von gut 50 Jahren Tausende von Kindern missbraucht zu haben.

\section{Erwartungen an die Medizin im Kontext sexuellen Missbrauchs: Diagnose, Klassifikation, Therapie}

Der medizinische Kinderschutz begann mit dem Blick auf Röntgenbilder. Ausgangspunkt war die pädiatrische Radiologie (z.B. Caffey 1946). C. Henry Kempe gründete 1958 in Denver ein „Child Protection Team“ und veröffentlichte 1962 den wegweisenden Artikel „The Battered-Child Syndrome“. 1968 gaben Kempe 
und Helfer das bis heute als Standardwerk geltende Buch "The Battered Child“ zum ersten Mal heraus. 1977 wurde das internationale Journal Child Abuse \& Neglect gegründet, welches interdisziplinär den wissenschaftlichen Blick auf Kindesmisshandlung voranbrachte. Rechtsmedizinische Autorinnen in Deutschland, darunter die Pionierin Elisabeth Trube-Becker, wiesen aufgrund ihrer Befunde auf Gewalt gegen Kinder hin.

Die öffentliche Debatte über sexuellen Missbrauch in der Bundesrepublik begann mit der Veröffentlichung des Buches von Barbara Kavemann und Ingrid Lohstöter „Väter als Täter - sexuelle Gewalt gegen Mädchen“ im Jahr 1984. An die Medizin richtete sich die Erwartung, bei sexuellem Missbrauch Symptomkomplexe und Syndrome zu beschreiben. Diese sollten es ermöglichen, sexuellen Missbrauch objektiv zu diagnostizieren, ohne mit dem Patienten sprechen zu müssen. Das Vorgehen aus der pädiatrischen Radiologie, wo bestimmte Frakturmuster als charakteristisch für Kindesmisshandlung erkannt wurden und Eltern dann mit den Befunden konfrontiert werden konnten, sollte - so der Wunsch oder vielleicht auch der Allmachtsglauben an die Möglichkeiten der Medizin - auch im medizinischen Umgang mit der Diagnostik bei sexualisierter Gewalt möglich sein.

Es entwickelte sich rasch eine Debatte über Checklisten und das sogenannte „sexual abuse syndrome“. ${ }^{2}$ Doch einzelne Symptome wie z.B. das Einnässen sind zu unspezifisch; ein zuverlässig erkennbares Verhaltenssyndrom des sexuellen Missbrauchs gibt es nicht. Viele Symptome sind altersabhängig und eben nicht spezifisch für sexuelle Übergriffe.

Auch die Hoffnung auf spezifische gynäkologische Befunde wurde bald relativiert. Enorme diagnostische Fortschritte brachte nur die Humangenetik mit sich. In Fällen, in denen Sperma am Körper oder an der Kleidung eines Kindes asserviert wird, kann nicht nur die Tat, sondern mit extrem hoher Sicherheit auch der spezifische Täter genetisch festgestellt werden. Allerdings werden die wenigsten Patientinnen und Patienten direkt nach einem sexuellen Übergriff vorgestellt. Bei den meisten findet sexueller Missbrauch chronisch statt, und die Betroffenen sprechen darüber häufig erst relativ spät, sei es gegenüber Vertrauenspersonen in ihrem Umfeld oder, wenn sie aufgrund ihrer Belastungen in Therapie kommen, erst nachdem sie Vertrauen zur Therapeutin oder zum Therapeuten gefasst haben. Nicht zuletzt aus Mangel, den Missbrauch eindeutig physiologisch identifizieren zu können, konzentrierte sich die Medizin zunächst auf Möglichkeiten der Klassifizierung.

2 Zusammenfassend Friedrich 1992, wo er auch seine Ergebnisse zu einer Verhaltensliste in Bezug auf sexualisiertes Verhalten beschreibt. Ich habe damals in der Praxis der Kinderpsychologie und Kinderpsychiatrie (Fegert 1987) kritisch Stellung genommen. 
In der frühen Debatte in der Bundesrepublik der 198oer Jahre konnte man schon an der Wortwahl erkennen, wer aus welcher Perspektive sich jeweils mit sexuellem Missbrauch auseinandergesetzt hatte. Die Bezeichnung "sexueller Missbrauch“ und später auch häufig „sexuelle Gewalt“ oder „sexualisierte Gewalt" lenkte den Blick auf Straftaten und auf Gewalt in Abhängigkeitsverhältnissen, also auf Beziehungstaten, zu denen Kinder schon aufgrund ihres Alters niemals eine informierte Zustimmung geben konnten. Die korrektere Bezeichnung „sexuelle Kindesmisshandlung“ („child abuse“ bedeutet „Kindesmisshandlung“ und „sexual abuse“ hätte eigentlich mit "sexuelle Kindesmisshandlung“ übersetzt werden müssen), die im medizinischen Bereich in Deutschland verbreitet war, ordnete sexuellen Missbrauch vor allem den unterschiedlichen Formen der Kindesmisshandlung und Vernachlässigung zu. In dem von Remschmidt, Schmidt und Poustka (2001) eingeführten multiaxialen Klassifikationssystem in der Kinder- und Jugendpsychiatrie wurde auf der Achse V (psychosoziale Belastungen) auch intrafamiliärer und extrafamiliärer sexueller Missbrauch in der klinischen Dokumentation regelmäßig erfasst. Mit der Einführung des internationalen Klassifikationssystems (Internationale Klassifikation von Krankheiten der Weltgesundheitsorganisation) ICD-10 standen auch Kodes zur Erfassung dieser Misshandlungsformen (wie „körperlicher“, „sexueller“ oder „psychischer Missbrauch“) zur Verfügung.

Allerdings war die Verwendung dieser Dokumentationsschlüssel in Deutschland lange tabu. Ärztinnen und Ärzte mussten befürchten, dass ihre Patienten auch gegen ihren Willen als Opferzeugen in Strafverfahren hineingezogen würden, wenn in amtlichen Dokumenten diese Klassifizierung auftauchte. Erst 2013 gelang es, im Sozialgesetzbuch (SGB) V (§ 294 a) eine Norm zu verändern, die bislang die Krankenkassen veranlasst hatte, bei Nennung solcher Merkmalschlüssel automatisch Strafanzeige gegen Unbekannt zu stellen.

Dadurch aber befand sich die Medizin bis vor wenigen Jahren in einem „Blindflug“ in Bezug auf die Häufigkeit einer Missbrauchs- oder Misshandlungsvorgeschichte bei Patienten in Krankenhausbehandlung. Systemisch orientierte Therapeuten sprachen in den 198oer Jahren oft (verharmlosend) von der "Inzestfamilie“ und setzten sich damit dem Vorwurf aus, bei der Betrachtung von Familiensystemen veraltete Homöostasemodelle vor Augen zu haben, die Machtfragen und Fragen von Schuld und Verantwortung negierten und in Interdependenzen auflösten. Kinder waren in einer solchen Sicht oft Symptomträger eines dysfunktionalen Systems. In diesen Kontexten wurde häufig vermieden, von Tätern und Opfern zu sprechen.

Grundsätzlich ist Missbrauch keine Erkrankung an sich und wird auch nicht als solche klassifiziert. Sexueller Missbrauch ist eine potentiell traumatisierende Grenzerfahrung, die manche Kinder und Jugendlichen stark 
belasten kann, während andere scheinbar resilient trotz schwerer Übergriffe keine behandlungsbedürftigen Störungsbilder zeigen. In der Literatur geht man davon aus, dass etwa ein Viertel bis ein Drittel der Betroffenen wenigstens in den ersten Jahren nach dem Missbrauch in dem Sinne resilient reagiert und dass keine behandlungsbedürftige Störung diagnostiziert werden kann (Domhardt u.a. 2015; Witt u.a. 2014).

Gleichwohl ist das Risiko für spätere Folgeerkrankungen erhöht, insbesondere in Transitionsphasen wie der Pubertät, in der Kinder eher verstehen, was die sexuelle Komponente dieser Handlungen bedeutet. Diagnostiziert werden können also bei Kindern, die Missbrauch erlebt haben, kurzfristige Anpassungsstörungen, welche vorübergehend auftreten, dazu emotionale Störungen, Depression, Angst und insbesondere Vermeidung sozialer Kontakte, die häufig zu einer starken sozialen Beeinträchtigung führen, sowie emotional dysreguliertes, aggressives Verhalten. Manche Kinder und Jugendliche zeigen auch das Vollbild einer posttraumatischen Belastungsstörung, wobei gerade bei kleineren Kindern häufig nicht alle bei Erwachsenen üblichen Symptome auftreten, so dass die Diagnosekriterien trotz einer deutlichen Belastung der Kinder nicht erfüllt werden können.

Die internationale Klassifikation von Krankheiten ist derzeit in Überarbeitung. Die ICD-11, die bald auch in Deutschland eingeführt werden soll, wird eine wichtige Neuerung mit sich bringen, nämlich die Einführung einer diagnostischen Kategorie für Komplextraumatisierungen. Diese neue Diagnose beschreibt Menschen, die unglücklicherweise mehrere Belastungen, wie z.B. frühe Vernachlässigung, dann Kindesmisshandlung und auch sexuellen Missbrauch überstehen müssen.

In der Psychiatrie, der Kinder- und Jugendpsychiatrie und generell in der Medizin wird mit Diagnosen gearbeitet, um Störungen zu klassifizieren, die mit Krankheiten einhergehen. Die Feststellung einer Diagnose ist oft die Voraussetzung für sozialrechtliche Ansprüche auf Krankenbehandlung, insbesondere auch Psychotherapie und andere therapeutische Behandlungen. Der medizinisch diagnostische Blick und die Erfüllung einzelner Kriterien haben deshalb oft eine erhebliche sozialrechtliche Relevanz in Bezug auf den Zugang zu Hilfen. Dies trifft auch auf Hilfen zur Teilhabe zu, die über den $\S 35$ a SGB VIII im Kindes- und Jugendalter bei einer so genannten „drohenden seelischen Behinderung" gewährt werden.

Dabei ist die Diagnose einer psychischen Störung nach dem jeweils geltenden Klassifikationssystem der WHO eine notwendige, aber nicht hinreichende Voraussetzung für die Hilfegewährung bei Teilhabebeeinträchtigung durch psychische Störungen. Tatsächlich muss im Einzelfall neben der heilberuflichen Diagnose unter Federführung der Jugendhilfe mit Beteiligung der 
Betroffenen und ihrer Sorgeberechtigten im Rahmen einer Hilfeplanung das Ausmaß der Teilhabebeeinträchtigung festgestellt werden.

Traumadiagnosen waren und sind immer auch politisch umstritten, denn an ihnen machen sich, z.B. bei Kriegsveteranen oder Opfern von Terroranschlägen, Ansprüche auf Hilfen oder Rentenzahlungen fest. Insofern hat die Festlegung von Symptomschwellen und Diagnosekriterien immer auch eine sozialpolitische Bedeutung. Es war über Jahrzehnte auffällig, dass in dem amerikanischen Klassifikationssystem, dem DSM, fast alle anderen Diagnosekriterien für andere Störungsbilder leichter erfüllt werden als in der internationalen Klassifikation der Erkrankungen. Damit sind medikamentöse Behandlungen früher möglich, was zu deutlich mehr Medikamentenverschreibungen führt. Die Diagnose „posttraumatische Belastungsstörungen“ hatte in den USA bislang immer eine höhere Schwelle als in der ICD. Dies hatte sicher auch damit zu tun, dass die frühe Forschung zu posttraumatischen Belastungsstörungen vor allem mit der Behandlung von Vietnam-Veteranen und deren Ansprüchen gegenüber der amerikanischen Regierung verbunden war.

Für den medizinischen Blick auf Opfer bedeutet dies, dass Diagnosen von Traumafolgestörungen nicht in Stein gemeißelte Entitäten sind, sondern Setzungen von Expertengremien, die aufgrund empirischer Untersuchungen die Grenze zwischen "noch normal“ und "nicht mehr normal“ definieren. Je nachdem wie diese Grenze gezogen wird, können sich Häufigkeiten erheblich unterscheiden - und in der Folge auch Rechtsansprüche in Bezug auf Hilfeleistungen.

Neben der Kerndiagnose „Posttraumatische Belastungsstörung“ werden andere kurzfristige, mittelfristige und langfristige Traumafolgestörungen beschrieben. Als Leiter einer der größten und einflussreichsten Studien im Bereich Public Health untersuchte der Internist Vincent Felitti mit seinem Team 17337 Teilnehmende aus der amerikanischen Mittelklasse in einer Versichertenpopulation als Teil einer routinemäßigen Gesundheitsuntersuchung und fragte nach zehn belastenden Lebensereignissen, den fünf Misshandlungsformen (emotionale Misshandlung, körperliche Misshandlung, sexueller Missbrauch, emotionale Vernachlässigung, körperliche Vernachlässigung) und nach fünf Typen von Problemen im Elternhaus (household dysfunction): Elterliche Scheidung/Trennung, Gewalt gegen die (Stief-)Mutter, Substanzmissbrauch im Haushalt, psychische Störungen im Haushalt, Inhaftierung eines Familienmitglieds.

$5^{1} \%$ der Befragten hatten mehr als eine Kategorie belastender Kindheitsereignisse erlebt, $6,2 \%$ berichteten mehr als 4 solcher ACE (Adverse Childhood Experiences). Mit der Anzahl von ACEs stieg das Risiko für eine Vielzahl von 
physischen und psychischen Erkrankungen. Felitti schloss daraus: „Much of what is recognized as common in adult medicine, is the result of what is not recognized in childhood" (Felitti 2002).

Heute dient die sogenannte „ACE-Pyramide“ als konzeptuelles Gerüst für das Verständnis von Langzeitwirkungen über die Lebensspanne: Negative Kindheitserfahrungen führen $\mathrm{zu}$ einer negativen Beeinflussung der neurobehavioralen- und Persönlichkeitsentwicklung und damit zu sozialen, emotionalen und kognitiven Beeinträchtigungen. Diese Dispositionen begünstigen die Aufnahme von gesundheitlichem Risikoverhalten, insbesondere im Umgang mit Spannungszuständen, wie Substanzkonsum, süchtiges Essverhalten, selbstverletzendes Verhalten. Über diese Gesundheitsrisiken kann ein sogenanntes „metabolisches Syndrom“ mit körperlicher Krankheit, Behinderung und sozialen Problemen entstehen. Letztendlich steigt das Risiko für einen früheren Tod.

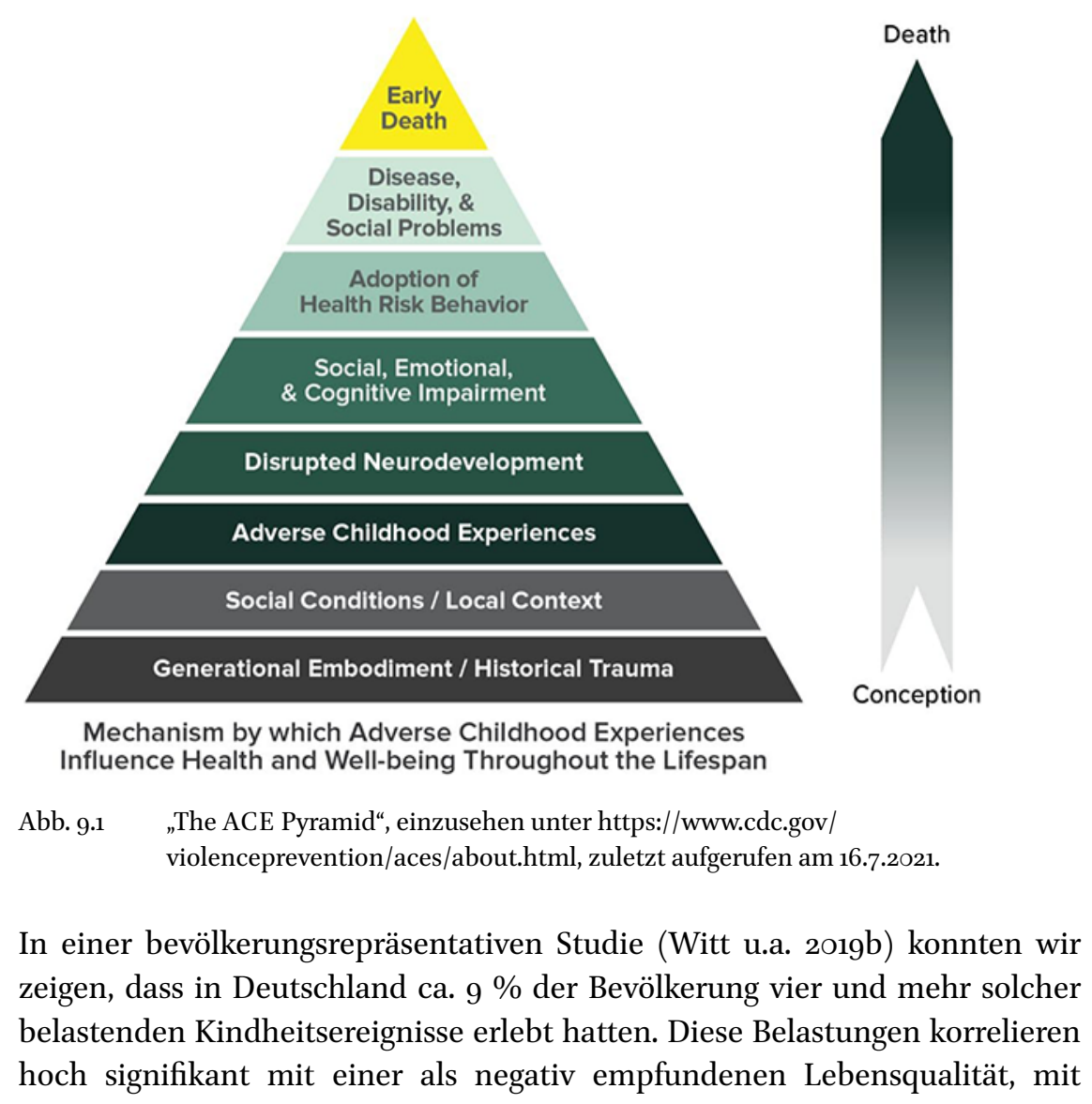


höheren Risiken für Angst und Depression, aber auch Aggressivität. Personen mit mehr als vier ACEs hatten in den letzten 12 Monaten mehr als viermal so häufig Personen verbal angegriffen oder herabgesetzt und mehr als zehnmal so häufig Personen körperlich angegriffen. Dies hat Auswirkungen auf den Beziehungsstatus, so dass ein deutlich geringerer Teil dieser Personen in einer Partnerschaft lebt.

Auf der Basis des Modells von Felitti analysierten wir in einem deutschen Datensatz der Barmer Ersatzkasse zusammen mit den Kieler Gesundheitsökonomen in der Deutschen Traumafolgekostenstudie (Habetha u.a. 2012a; 2012b) die möglichen tangiblen Kosten der Traumatisierung, das heißt Gesundheitskosten, Kosten der Kinder- und Jugendhilfe, Ausbildungsförderung, Wertschöpfungsverlust etc. In einem moderaten Modell ermittelten wir jährliche Kosten für die deutsche Gesellschaft durch Folgen von Kindesmisshandlung/ Missbrauch und Vernachlässigung von elf Milliarden Euro.

\section{Der skeptische Blick auf Aussagemöglichkeiten von Kindern, die Debatte um Missbrauch mit dem Missbrauch und das so genannte „False Memory Syndrom“}

Gelingt es in rechtsmedizinischen oder kriminalistischen Untersuchungen nicht, eindeutige Spuren festzuhalten, die wie Sperma den genetischen Fingerabdruck des Täters enthalten, oder Videos von Gewalt gegen Kinder, die viele Täter anfertigen und wie Ware verbreiten, bleibt die Aussage der Betroffenen der Königsweg bei der Wahrnehmung von sexualisierter Gewalt gegen Kinder. Hier steht dann nicht nur oft Aussage gegen Aussage. Im Strafrecht darf der Beschuldigte schweigen, während kindliche Opferzeugen aussagen müssen, es sei denn sie hätten aufgrund von Verwandtschaftsverhältnissen ein Aussageverweigerungsrecht. Insofern hat sich der entwicklungspsychologische und klinische Blick auch schon früh mit den Aussagemöglichkeiten von Kindern beschäftigt.

Historisch war Glaubwürdigkeit und die Fähigkeit Zeugnis abzulegen an den Status des männlichen Bürgers gebunden. Aussagen von Frauen und Kindern wurden per se geringer gewertet. Lange wurde Glaubwürdigkeit als Persönlichkeitsmerkmal angesehen, und man versuchte, Opfer durch Beschreibung ihres "liederlichen Lebenswandels“ oder ihrer vermeintlich "aufreizenden Kleidung“ zu diskreditieren und ihnen eine Mitschuld an den erlebten Taten zu geben.

Der modernen Aussagepsychologie, die weitgehend ein deutscher Sonderweg geblieben ist, kommt das Verdienst zu, gezeigt zu haben, dass Glaubhaftigkeit in der Regel ein situatives Charakteristikum ist. Wir alle sind in 
bestimmten Situationen mit unseren Aussagen nur wenig glaubhaft, z.B. wenn eine Politesse uns fragt, wie lange wir hier schon geparkt haben. Hier neigen dann auch sonst wahrheitsliebende Menschen dazu zu flunkern. Wenn es aber um existenzielle Bedrohungen und Eindrücke geht, sind viele Menschen eher zurückhaltend und trauen sich lange nicht, darüber zu sprechen, oder haben gute Gründe, weshalb sie nach einigen gescheiterten Mitteilungsversuchen nicht mehr darüber sprechen wollen (Kavemann u.a. 2016). Der Rechtsgrundsatz "in dubio pro reo" führt dazu, dass im Strafverfahren bei der Auseinandersetzung mit Aussagen von Opferzeuginnen und Opferzeugen besonders kritisch umgegangen wird. Der Bundesgerichtshof hat in Strafsachen in seinem Urteil aus dem Jahr 1999 (BGH 1 StR 618/98) die "Nullhypothese“ aufgestellt, wonach die Aussage des Kindes zunächst als unwahr zu gelten habe. Erst wenn sich anhand sehr spezifischer Aussagemerkmale nachweisen lässt, dass das Kind tatsächlich Dinge erlitten hat, die es sich nicht hat ausdenken können, wird die Anschuldigung des Kindes für glaubhaft gehalten. Diagnostisch wird deshalb in diesem Kontext vor allem Spezifität angestrebt, nicht Sensitivität.

Anders ist dies im zivilrechtlichen Kinderschutz. Dort geht es im Zweifel nicht darum, definitiv zu wissen, wer zu welchem Zeitpunkt genau was getan hat, sondern darum, prognostisch die Bedrohung der Entwicklung eines Kindes einzuschätzen. Medizinische und psychologische Tests lassen sich durch Testgütekriterien charakterisieren. Die Debatte um das SARS-CoV2-Virus hat auch die Allgemeinbevölkerung damit vertrauter gemacht, dass allgemein übliche Tests auch Irrtumswahrscheinlichkeiten haben. Die Wahrscheinlichkeiten lassen sich statistisch bestimmen. Nur bei wenigen Tests liegen gleichzeitig eine extrem hohe Sensitivität und Spezifität vor wie bei den heute gebräuchlichen AIDS-Tests oder jetzt auch den Schnelltests im Kontext SARS-CoV-2, die sich durch eine hohe Treffsicherheit auszeichnen. Das ist aber nicht die Regel in der Medizin oder der Psychodiagnostik.

Bei der so genannten „Glaubhaftigkeitsbegutachtung" handelt es sich nicht einmal um ein hypothesenüberprüfendes Verfahren mit entsprechenden Testgütekriterien, sondern um eine qualitative, kriterienbasierte Beurteilung von Textmaterial, d.h. der Aussage der Betroffenen. Diese qualitative Analyse erfolgt entsprechend der Unschuldsvermutung primär unter dem Blickwinkel, ob die vom betroffenen Kind gemachte Aussage auch ohne ein reales Taterleben, z.B. durch suggestive Einflüsse wie etwa durch Beobachtungen aus Videos, hätte zustande kommen können.

Dies führt dazu, dass Zweifel an der Aussage bleiben, insbesondere wenn sich Widersprüche z.B. in Aussagedetails zwischen Erstaussage bei der Polizei und späteren Vernehmungen ergeben haben oder wenn möglichweise suggestive Einflüsse oder das Ansehen von Videos nicht ausgeschlossen werden können. 
Häufig werden Aussagemängel gerade durch die befragenden Personen herbeigeführt, indem Dinge gefragt werden, die Kinder in einem gewissen Alter noch nicht erfassen können oder in denen ein zu hoher Befragungsdruck dazu führt, dass Kinder konfabulieren, um die erwachsene Autoritätsperson respektvoll zufrieden zu stellen.

Man muss bedenken, dass wir alle in der Schule gelernt haben, dass es eine sehr ungeschickte Strategie ist zu schweigen, wenn man von Lehrern gefragt wird. Es empfiehlt sich eher etwas zu sagen und dann aus der Reaktion zu schließen, ob man ungefähr richtig liegt. Kindern muss deshalb im Rahmen einer Begutachtung deutlich gemacht werden, dass es sich nicht um eine Prüfungssituation handelt, in der Antworten bewertet werden und NichtWissen als schlechte Leistung angesehen wird, sondern dass es explizit erlaubt ist, sich nicht zu erinnern und dass es erwünscht ist, nur das sicher Erinnerte $\mathrm{zu}$ berichten. Entwicklungspsychologisch und entwicklungspsychopathologisch liegen zahlreiche Erkenntnisse zur kindgerechten Befragung vor (vgl. Niehaus/Volbert/Fegert 2017), allerdings werden diese in der Praxis viel zu wenig berücksichtigt.

In vielen Ländern Europas wird derzeit versucht, Kinder unter möglichst idealen Bedingungen nur einmal zu vernehmen und diese Aussagen sowohl im strafrechtlichen wie im zivilrechtlichen Kontext und zur Organisation von entsprechenden Hilfen zu verwenden. Die Einmalvernehmung mit Videoaufzeichnung ist z.B. in der Schweiz die Regel. In Skandinavien setzt sich die Barnahus-Bewegung immer stärker durch, wo in entsprechenden Häusern die Diagnostik, die Hilfeplanung und die Vernehmung für die Strafverfolgung rund um das Kind organisiert werden können (vgl. Fegert u.a. 2016).

In den 199oer Jahren war es im Kontext verschiedener großer Strafverfahren, die letztendlich mit dem Freispruch der Angeklagten endeten, weil eine so genannte „Aufdeckungsarbeit“ manipulativ mit Kindern umging und wohl suggestiv Aussagen induziert hatte, generell zu der Debatte gekommen, dass häufig Missbrauch mit dem Missbrauchsvorwurf getrieben werde. Familiengerichtliche Gutachter behaupteten, dass der falsche Missbrauchsvorwurf in Scheidungsverfahren eine große Rolle spiele. Spätere Forschungsprojekte zeigten allerdings, dass sich diese Annahmen nicht bestätigen ließen (Busse/ Volbert/Steller 1996).

Charakteristisch für diese Zeit, in der wir ein Projekt mit prospektiver Begleitung sexuell missbrauchter Kinder in verschiedenen Kontexten, also mit Beratungsstellen, im Strafverfahren und in zivilrechtlichen Verfahren, durchführten und gleichzeitig Interviews mit allen Akteuren führten, waren eine gewisse Sprachverwirrung und Konfusion in Bezug auf die Zuständigkeiten. Strafverfolger sprachen häufig davon, dass sie Kindern helfen wollten, und 
hatten sich auch sehr darum bemüht, kindgerechte Vernehmungsbedingungen zu schaffen. Konkret hatten sie aber oft keinen Überblick über mögliche Hilfen und Beratungsstellen. Stattdessen war die von dem deutschen Kinderund Jugendpsychiater Fürniss (1991; 1993) propagierte Aufdeckungsarbeit gebräuchlich, und man versuchte sich mehrheitlich in Ermittlungstätigkeiten.

Gleichzeitig wurde eine Debatte über die Belastung von Kindern in gerichtlichen Verfahren geführt. Schon 1983 hatte der deutsche Kinder- und Jugendpsychiater Reinhart Lempp von der Gefahr der sekundären Viktimisierung durch Verfahren gesprochen und damit ein Diktum von Karl Kraus aufgegriffen, der gemeint hatte, dass die juristische Abwicklung dieser Taten oft den Schaden mehre, den die Tat bewirkt habe. Gerade in komplexen, fortgesetzten Missbrauchsfällen etablierte sich mehr und mehr eine Tendenz, von einer Strafanzeige abzusehen, um Kindern die darauffolgenden Belastungen zu ersparen (Fegert u.a. 2001).

Im Kontext von Strafverfahren wurden in dieser Zeit zunehmend Opferschutzmaßnahmen, Videovernehmungen, Zeugenbegleitung etc. ermöglicht. Allerdings werden Opferzeugen diese Unterstützungsangebote heute viel zu selten angeboten. Insofern ist die Forderung des Europarats nach „child friendly justice“ noch lange nicht eingelöst. Häufig sind Kinder auch heute Objekte in Verfahren und nicht Rechtssubjekte. So wurden z.B. im Missbrauchsfall von Lügde, wo zahlreiche Kinder in einem Wohnwagen auf einem Campingplatz über Jahre hinweg missbraucht wurden, die Eltern von der Polizei ohne Rechtsgrundlage aufgefordert, ihre Kinder, die zum Teil massive Belastungssymptome zeigten, nicht in Beratung oder Krankenbehandlung zu bringen, damit die Aussage für das Strafverfahren nicht verfälscht werde. Hier werden Kinder primär als Beweismittel und nicht als schutz- und hilfebedürftige Menschen angesehen.

Auch in kirchenrechtlichen Verfahren haben Betroffene keinen eigenen Rechtsstatus. Opferunterstützende Maßnahmen wie etwa das Recht auf Nebenklagevertretung etc., existieren nicht. Das Kirchenrecht ist auch dieser Stelle ein reines Klerikerrecht, bei dem es um Verstöße gegen das sechste Gebot geht. Dabei bedient man sich mitunter durchaus des medizinisch/gerichtspsychologisch kritischen Blicks auf Kinderaussagen und Betroffenenaussagen. Über den Fortgang der Verfahren erfahren diese aber nichts. Dies ist ein deutlicher Verstoß gegen die Kinderrechtskonvention der Vereinten Nationen, welche Kindern in allen sie betreffenden Verfahren Informationsrechte zusichert und die auch vom Vatikan ratifiziert wurde.

Vergleicht man also das Kirchenrecht mit dem Standesrecht in den Heilberufen, dann fällt auf, dass es im Standesrecht primär um Garantenpflichten geht. Der alte römische Rechtsgrundsatz salus aegroti suprema lex, wonach das 
Heil des Kranken das höchste Rechtsgut sei, bestimmt das ärztliche Standesrecht bis heute. In Bezug auf sexuelle Abstinenz gegenüber Klienten lassen sich diese Vorschriften auf den Eid des Hippokrates zuführen: „In welches Haus immer ich eintrete, eintreten werde ich zum Nutzen des Kranken, frei von jedem willkürlichen Unrecht und jeder Schädigung und den Werken der Lust aus den Leibern von Frauen und Männern, Freien und Sklaven" (aus dem Eid des Hippokrates in der Übersetzung von Karl Deichgräber). Im Gegensatz zum Strafrecht, wo der Zweifelsgrundsatz der zentrale bestimmende Maßstab ist, gilt also im Standesrecht eine höhere Sensitivität. Es geht um die Eignung und auch die herausgehobene, privilegierte Position des Arztes/der Ärztin im Umgang mit Patientinnen und Patienten. Um eine Gefährdung von Patientinnen und Patienten zu vermeiden, kann im Standesrecht die Berufsausübung teilweise oder ganz untersagt werden, befristet oder unbefristet, je nach Anlass der Untersagung. Zu beachten ist, dass solche Untersagungen, z.B. bei suchtkranken Ärztinnen und Ärzten, weitergehende Sanktionen ermöglichen, die auch dann verhängt werden können, wenn das Strafrecht keine Handhabe bietet. Allerdings muss man auch in Bezug auf das heilberufliche Standesrecht feststellen, dass es keine ausformulierten Rechtspositionen für die Betroffenen gibt.

\section{Zum Wandel des psychiatrischen Blicks seit der Jahrtausendwende}

In den USA waren "9/11“ und der Hurrikan „Katrina“ 2005 Ausgangspunkte einer veränderten Wahrnehmung und Bewertung von Traumatisierung. Ein nationales Traumforschungsnetzwerk wurde gegründet. Neue Methoden in der Hirnforschung, insbesondere der funktionellen Bildgebung (MagnetResonanz-Tomographie, MRT), ermöglichten zudem ein besseres Verständnis von Traumagedächtnis und posttraumatischer Symptomatik (Fegert/Plener 2016). Dies führte auch zu einer veränderten Einschätzung der Glaubhaftigkeit retrospektiver Erinnerungen, etwa wenn Patienten, die angaben, sexuellen Missbrauch erlebt zu haben, in funktioneller Bildgebung in den Hirnarealen, die für die Genitalregion zuständig sind, sich von Nicht-Betroffenen und auf andere Weise misshandelten Personen unterschieden. Die Studien der Nemeroff-Gruppe in Deutschland (2013; Heim/Nemeroff 2001) haben gruppenstatistisch eine quasi topografische Zuordnung in funktionellen MRTs zu einzelnen Misshandlungsformen zeigen können. Der medizinische Blick ist dann nicht nur eine Metapher. In den aufsummierten Bildbefunden von Betroffenen sieht man im Vergleich zu Kontrollprobanden Unterschiede in entsprechenden Gehirnstrukturen. Der Missbrauch ist zum sichtbaren 
Substrat geworden und wird deshalb - vielleicht weil er mit extrem teuren, hochkomplexen Geräten wenigstens im gruppenstatistischen Vergleich in seinen Folgen sichtbar gemacht werden kann - als Realität anerkannt. Damit scheint zwar nicht im Einzelfall, aber wenigstens gruppenstatistisch eine sichtbare Beweisbarkeit sexuellen Missbrauchs möglich zu sein, die ähnlich wie charakteristische Röntgenbefunde bei der körperlichen Misshandlung, sichtbare Belege liefern, ohne dass man mit den Betroffenen reden oder den Opfern glauben muss.

Die starken Belastungen, die Übererregbarkeit (hyperarousal) und die Veränderung der Stressregulation bei Betroffenen wurden nun besser verstanden. Und es waren gerade diese biologischen Korrelate, welche die Einschätzungen in der Psychiatrie und Kinder- und Jugendpsychiatrie veränderten, weil nun neurobiologische und neuropsychologische Befunde ohne interferierende subjektive Bewertung durch Patientinnen und Patienten scheinbar objektiv Unterschiede aufzeigten.

Nicht zuletzt auf der Basis dieser Befunde wurde eine neue Therapie entwickelt. Diese gründete auf einer Konfrontation des Traumatisierten mit der angstauslösenden Erinnerung. Die traumafokussierte, kognitive Verhaltenstherapie für Kinder und Jugendliche ist mittlerweile die weltweit bestuntersuchte Therapiemethode mit hoher Evidenz für ihre Wirksamkeit. Doch auch andere Therapien wie die narrative Expositionstherapie oder EMDR (Eye Movement Desensitization and Reprocessing) basieren auf der Exposition gegenüber einem Narrativ. Dabei wird gelernt, dass es aushaltbar ist zu berichten, was geschehen ist. Man kann das Geschehene nicht ungeschehen machen, aber man kann es biografisch einordnen und dann auch nach vorne blicken. So entwickelte sich nach dem Massaker auf Utøya in Norwegen ein vorbildliches Kindertrauma-Interventionsnetzwerk. Leider waren es bislang nur solche schrecklichen Erfahrungen, die Staaten veranlasst haben, das Thema „Traumatisierung“ ernst zu nehmen - und das immer zu spät.

Bei der Reform des Sozialen Entschädigungsrechts 2020 durch den Deutschen Bundestag haben die Bundesländer verhindert, dass spezifische Kindertrauma-Ambulanzen eigens erwähnt wurden. Die Begründung lautete, es gebe nicht genügend ausgebildete Therapeuten und auch keine Ressourcen. Veränderungen im medizinischen Wissen und medizinischen Blick allein reichen anscheinend nach wie vor nicht aus, wenn sich nicht auch das gesellschaftliche Verständnis verändert.

Das große Problem ist daher aktuell die flächendeckende Verfügbarkeit erfolgversprechender Therapieansätze. Derzeit kann aber davon ausgegangen werden, dass Traumafolgestörungen gut untersucht sind und auch relativ gut 
durch entsprechende spezifische psychotherapeutische Methoden behandelbar sind. Medikamente sind, wenn überhaupt, nur in Ausnahmefällen unterstützend einzusetzen.

Während also die Psychotherapie posttraumatischer Belastungsstörungen heute weitgehend zum gesicherten Wissen gehört, gibt es aus medizinischer Sicht noch große Verständnis- und Forschungslücken in Bezug auf die oben dargestellten Zusammenhänge zwischen belastenden Kindheitsereignissen und späteren körperlichen und psychischen Langzeitfolgen. In einer interdisziplinären Perspektive auf multidimensionale Traumawissenschaften versuchen wir in einem im Bau befindlichen Traumaforschungsgebäude Pathomechanismen zu erforschen, welche von den erlebten Belastungen über entzündliche Prozesse, Hormone wie Cortisol und Oxytocin, oder auch über Transmitterstoffe wie Endocannabinoide und Katecholamine zu teilweise bleibenden Veränderungen geführt haben. Hier verlässt also die Traumaforschung erneut ihren Sonderweg des Hörens und Sprechens und gliedert sich wieder in die derzeit prestigeträchtigsten Methoden des medizinischen Blicks ein: die Untersuchung der molekularen Vorgänge im Körper und Gehirn vor dem Hintergrund genetischer Prädispositionen und diverser sogenannter „Umweltbelastungen“.

\section{Das „neue Hören“: 2010 als Zäsur im Umgang mit Betroffenen/ Opfern}

Im Januar 2010 kam der sogenannte „Missbrauchsskandal in Institutionen“ letztendlich dadurch in Gang, dass Betroffene redeten und sich an die Institution wandten, in der sie missbraucht worden waren. Eine Einzelperson in verantwortlicher Leitungsposition, nämlich Pater Klaus Mertes SJ am Berliner Canisius-Kolleg, schenkte diesen Betroffenen Gehör und nahm ihre Aussagen ernst. Betroffene traten selbst vor die Presse, was wiederum mit Angst besetzt war. „Es bedeutet einen Kontrollverlust, und nichts fürchten Opfer mehr, als einer solchen Situation erneut ausgesetzt zu sein", so berichtet Matthias Katsch aus eigener Erfahrung in seinem 2020 veröffentlichten Bericht. Dabei problematisierte er die Begriffe, mit denen sich die Opfer sexuellen Missbrauchs selbst bezeichneten, schließlich wollten sie nicht auf den Opferstatus reduziert werden. „Opfer waren wir gewesen, ja, aber jetzt wollten wir nicht länger als hilflos und ausgeliefert erscheinen." (Katsch 2020).

Die allgemeine Bereitschaft, die Stimmen der Betroffenen zu hören und sie ernstzunehmen, veränderte auch die Medizin, die lange das von Betroffenen Gehörte negiert oder in Phantasien umgedeutet hatte. Im Frühjahr 2010 
richtete die katholische Kirche eine Hotline ein, um Betroffenen Gehör zu schenken, organisiert vom späteren Präventionsbeauftragten des Bistums Trier, Dr. Andreas Zimmer (2014), mit dem ich damals in Kontakt stand. Gleichzeitig wurde die ehemalige Bundesfamilienministerin Dr. Christine Bergmann vom Bundeskabinett zur ersten Unabhängigen Beauftragen für Fragen des sexuellen Kindesmissbrauchs ernannt. Kurz nach der Ernennung bat sie mich, den Aufbau einer Anlaufstelle für Betroffene konzeptionell und wissenschaftlich zu begleiten.

So entstand der weltweit bislang größte Prozess, in dem Betroffenen systematisch Gehör geschenkt wurde. Mehr als 20 ooo Personen wandten sich innerhalb eines Jahres an diese telefonische Anlaufstelle, etwa 7 ooo gestatten uns, ihre Angaben für Forschungszwecke auszuwerten (Fegert u.a. 2013; Rassenhofer u.a. 2013; 2015). Die Ergebnisse in Bezug auf die Belastungen im weiteren Leben waren vergleichbar mit dem, was sich in der katholischen Hotline zeigte. Nach längeren diplomatischen Aktivitäten gelang es uns sogar, beide Datensätze, die mit vergleichbarer Methodik erhoben worden waren, in Ulm wissenschaftlich auszuwerten (Fegert u.a. 2013; Rassenhofer u.a. 2015).

Das methodische Vorgehen lag damals darin, die Betroffenen, die anriefen, frei erzählen zu lassen, also nicht suggestiv nachzufragen, sondern sie einfach berichten zu lassen und ihnen zuzuhören. Die einzigen Fragen, die konkret gestellt wurden, betrafen „Forderungen an die Politik“ und „Wünsche für die Zukunft“. Eine der häufigsten Forderungen war der altruistische Wunsch für die kommenden Generationen, dass heutigen und künftigen Kindern mehr zugehört und früher geglaubt werde sowie mehr Unterstützung und Hilfe. Wir hörten von zahllosen Irrwegen durch das Hilfesystem, von Professionellen und Familienangehörigen, die Betroffenen nicht glaubten, von Geschwistern, die den Kontakt abgebrochen hatten, und von jahrelangen Gerichtsverfahren, die mit Freisprüchen für die Täter endeten. Eine weitere zentrale Forderung war mehr Prävention, um heutigen Kindern einen langen Leidensweg zu ersparen und mehr Information für alle Fachkräfte, die mit Kindern arbeiten.

\section{Fazit}

Für die Prävention sexualisierter Gewalt an Kindern ist eine Kultur des Hinsehens unabdingbar. Hinsehen hilft beim Erkennen von Strukturen, beim Ausleuchten der Dunkelräume durch Analysen von Akten und Verwaltungshandeln, beim Nachvollziehen der Versetzungspraxis und bei der Diagnostik von psychischen Folgen, und es hilft vor allem bei der Strafverfolgung pornographischer Gewalt gegen Kinder im Internet. Wenn man hier hinsieht, merkt 
man, warum auch der Besitz von Videos, die Verbrechen sexualisierter Gewalt an Kindern wiedergeben, nicht mit dem Konsum von Pornographie zu vergleichen ist, die von bezahlten Darstellern zur Unterhaltung und sexuellen Stimulation massenhaft hergestellt wird.

Der Begriff "Kinderpornographie“ verharmlost in dieser Analogie das Hinsehen auf Gewalthandlungen, eben nicht bloß Gewaltdarstellungen. Hinzu kommt, dass oft Geldgeber in Netzwerken Regie führen und von den direkt die Kinder ausbeutenden und vergewaltigenden Personen in Echtzeit bestimmte Handlungen gegen Geld verlangen. Ein differenzierter Blick täte hier gut. Für viele Betroffene ist aber die Tatsache, dass diese Bilder häufig nie mehr zum Verschwinden zu bringen sind, eine weitere Quelle der Scham und der dauerhaften Bloßstellung.

Das zentrale (kinder- und jugend)psychiatrische Mittel zur Unterstützung Betroffener ist das fachliche Hinhören, das Sprechen über Geschehenes, das Einordnen. Betroffene wollen und sollen Gehör finden. Dazu müssen sie in die Lage versetzt werden, das Erlebte, das sie nicht ungeschehen machen können, zu berichten.

Alle erfolgreichen Therapieformen im Traumabereich bauen im weitesten Sinne auf einer Exposition gegenüber einem Narrativ der (schlimmsten) Erlebnisse auf. Eine biographische Einordnung ist ebenso wichtig wie ein Verständnis der an sich selbst erlebten Symptome, zum Beispiel bei posttraumatischen Belastungsstörungen, durch eine gute Psychoedukation.

Detailverliebtes Zuhören kann eine voyeuristische Komponente haben, aber eben um diese Details geht es in der Therapie nicht. Das kriminologisch notwendige Interesse an Details von Handlungen und der Abklärung einzelner Taten in ihren Abläufen ist für das psychiatrisch therapeutische Handeln irrelevant. Insofern macht "hören, verstehen, einordnen und Position beziehen" das psychiatrische Handwerkszeug im Umgang mit Betroffenen aus. Wichtig ist die Ermutigung zur Teilhabe.

Das Sehen der Situation allein bringt nichts. Vielmehr ist es wichtig, das biographisch Erlebte einordnen zu können, um jetzt, trotz dieser Erfahrungen, ein gutes Leben führen zu können. „A good life despite trauma“ ist deshalb auch der Claim der Deutschen Traumastiftung, deren Präsident ich bin. „Dazugehören“ kann über Gehör finden beginnen. Es waren die Erzählungen tausender Betroffener, die nach der Einrichtung des Runden Tischs sexueller Missbrauch und dem Aufbau der Anlaufstelle der ersten Unabhängigen Beauftragten als Handelnde die Agenda der Politik mitbestimmten. Zunächst geschah dies über die wissenschaftliche Erfassung, die Aggregation und die Wiedergabe ihrer Einzelstimmen, später endlich direkt durch Einbezug und die Bildung 
entsprechender Gremien wie z.B. des Betroffenenrats beim Unabhängigen Beauftragten für Fragen des sexuellen Kindesmissbrauchs.

Der medizinische Blick auf belastende Kindheitsereignisse und auf Misshandlungsformen wie sexueller Missbrauch in der Familie, im familiären Nahfeld oder in Institutionen hat sich durch gruppenstatistische Analysen retrospektiver Befragungen von Betroffenen, durch prospektive Langzeitstudien wie die Dunedin-Studie (Fergusson u.a. 2011) und durch die moderne Bildgebung sowie die Untersuchung anderer biologischer Korrelate deutlich verändert. Diese verschiedenen Untersuchungen belegen ein höheres Risiko für Opfer sexuellen Missbrauchs, im weiteren Leben psychische und physische Probleme zu bekommen, zeigen spezifische Benachteiligungen in der Teilhabe auf und weisen sogar auf das erhöhte Risiko der transgenerationalen Weitergabe belastender Erziehungserfahrungen hin.

Gruppenstatistik definiert die Leitplanken, auch in der Analyse therapeutischer Methoden und Zugangswege, und gibt uns Gewissheit in Bezug auf die generelle Wirksamkeit der heute in klinischen Leitlinien erwähnten Methoden. Der Blick der Gruppenstatistik trägt zum Verständnis und zur Einordnung bei, beschreibt aber nur relative Risiken, denn jeder Fall ist anders. Bis heute ist eine Individualdiagnostik mit Laboruntersuchungen oder neuropsychologischen Untersuchungen, die stattgefundenen sexuellen Missbrauch beweisen, kaum möglich, sieht man einmal von Situationen ab, in denen genetisches Material vom Täter asserviert werden konnte.

Das klinische Handeln ist deshalb auch heute im Einzelfall von Hören und Verstehen geprägt. Die Bereitschaft zu hören hat sich aber durch die wissenschaftlichen Ergebnisse in den Life-Sciences erheblich verändert, und das methodische Wissen über individuelle Traumatherapien und die zentrale Bedeutung eines Traumanarrativs kann als große Errungenschaft der vergangenen beiden Jahrzehnte bezeichnet werden. Umso wichtiger ist es, dass diese Erkenntnisse in der Breite Patienten zugänglich gemacht werden, so dass diese jederzeit die bestmögliche Therapie erhalten. Dringend notwendig erscheint eine wissenschaftliche Untersuchung darüber, ob Frühinterventionen und Psychotherapien tatsächlich, wie häufig im Strafrecht angenommen, die Aussagen von Kindern und Jugendlichen sowie erwachsenen Betroffenen verfälschen.

Alle heutigen empirischen Befunde zeigen, dass die Exposition gegenüber einem Traumanarrativ, also das Aushalten des Erzählens der erlebten Geschichte, das zentrale Element wirksamer Traumatherapien und Interventionen ist. Im Jahr 2015 konnten wir, angesichts des hohen Versorgungsdrucks geflüchteter junger Menschen in Deutschland, auch mit 
Gruppeninterventionen Erfahrungen sammeln und nachweisen, dass solche Gruppeninterventionen erfolgreich sein können (Pfeiffer u.a. 2018). Solche Beispiele können auch einen Hoffnungsschimmer für weniger gut therapeutisch versorgte Regionen auf der Welt sein, in denen Behandlungsmöglichkeiten für Kinder und Jugendliche fehlen, die von sexualisierter Gewalt oder organisierter sexueller Ausbeutung, insbesondere auch im Internet, betroffen sind.

Medizin kann das Erlebte nicht ungeschehen machen, aber es kann aushaltbar werden, das Erlebte auszusprechen. Betroffene können Gehör finden und Betroffene können auch in Bezug auf ihre Bedürfnisse nach Teilhabe anerkannt werden. Sprechen, nicht über Betroffene, sondern von Betroffenen und mit Betroffenen, ist ein zentrales Element des heutigen heilberuflichen Zugangs zur Thematik der sexualisierten Gewalt gegen Kinder und Jugendliche.

\section{Literaturverzeichnis:}

Busse, Detlef/Volbert, Renate/Steller, Max (1996): Belastungserleben von Kindern in Hauptverhandlungen. Abschlußbericht eines Forschungsprojekts im Auftrag des Bundesministeriums der Justiz. Bundesministerium der Justiz, Bonn.

Caffey, John (1946): Multiple fractures in the long bones of infants suffering from chronic subdural hematoma, in: Radiology 194, 163-173.

Domhardt, Matthias/Münzer, Annika/Fegert, Jörg M./Goldbeck, Lutz (2015): Resilience in Survivors of Child Sexual Abuse: A Systematic Review of the Literature, in: Trauma, Violence, \& Abuse 16(4), 476-493.

Dreßing, Harald/Salize, Hans J./Dölling, Dieter/Hermann, Dieter/Kruse, Andreas/ Schmitt, Eric/Bannenberg, Britta (2018): Forschungsprojekt: Sexueller Missbrauch an Minderjährigen durch katholische Priester, Diakone und männliche Ordensangehörige im Bereich der Deutschen Bischofskonferenz, 24.9., einzusehen unter https://www.dbk.de/fileadmin/redaktion/diverse_downloads/dossiers_2018/ MHG-Studie-gesamt.pdf, zuletzt aufgerufen am 9.4.2021.

Elliger, Tilman J./Schötensack, Kerstin (1991): Sexueller Mißbrauch von Kindern - eine kritische Bestandsaufnahme, in: Gerhardt Nissen (Hrsg.): Psychogene Psychosyndrome und ihre Therapie im Kindes- und Jugendalter. Bern: Hans Huber, 143-154. Fegert, Jörg M. (1987): Sexueller Mißbrauch von Kindern, in: Praxis der Kinderpsychologie und Kinderpsychiatrie 36(5), 164-170.

Fegert,Jörg M./Berger, Christina/Klopfer, Uta/Lehmkuhl, Ulrike/Lehmkuhl, Gerd (2001): Umgang mit sexuellem Missbrauch. Institutionelle und individuelle Reaktionen. Forschungsbericht. Münster: Votum.

Fegert, Jörg M./Rassenhofer, Miriam/Schneider, Thekla/Spröber, Nina/Seitz, Alexander (2013): Sexueller Kindesmissbrauch - Zeugnisse, Botschaften, Konsequenzen. 
Ergebnisse der Begleitforschung für die Anlaufstelle der Unabhängigen Beauftragten der Bundesregierung zur Aufarbeitung des sexuellen Kindesmissbrauchs, Frau Dr. Christine Bergmann. Weinheim/Basel: Beltz Juventa.

Fegert, Jörg M./Andresen, Sabine/Salgo, Ludwig/Walper, Sabine (2016): Hilfeangebote und strafrechtliche Fallbearbeitung bei sexueller Gewalt gegen Kinder - Vom Kind her denken und organisieren, in: ZKJ - Zeitschrift für Kindschaftsrecht und Jugendhilfe 11 (9/10), 324-334.

Fegert, Jörg M./Plener, Paul L. (2016): Auswirkungen von Gewalterfahrungen auf die Wahrnehmung und das Handeln betroffener Kinder, in: Sabine Völkl-Kernstock/ Christian Kienbacher (Hrsg.): Forensische Arbeit mit Kindern und Jugendlichen. Wien: Springer, 23-34.

Felitti, Vincent J. (2002): The relationship of adverse childhood experiences to adult health: Turning gold into lead, in: Zeitschrift für Psychosomatische Medizin und Psychotherapie 48(4), 359-369.

Fergusson, David M./Boden, Joseph M./Horwood, L. John/Miller, Allison L./Kennedy, Martin A. (2011): $M A O A$, abuse exposure and antisocial behaviour: 30-year longitudinal study, in: The British Journal of Psychiatry 198(6), 457-463.

Finkelhor, David (1979): Sexually Victimized Children. New York: Free Press.

Friedrich, William N./Grambsch, Patricia/Damon, Linda/Hewitt, Sandra K./Koverola, Catherine/Lang, Reuben A./Wolfe, Vicki/Broughton, Daniel (1992): Child Sexual Behavior Inventory: Normative and clinical comparisons, in: Psychological Assessment 4(3), 303-311.

Fürniss, Tilman (1991): The Multiprofessional Handbook of Child Sexual Abuse. London/New York: Routledge.

Fürniss, Tilman (1993): Verleugnungsarbeit, in: Gabriele Ramin (Hrsg.): Inzest und sexueller Missbrauch. Paderborn: Junfermann, 63-9o.

Habetha, Susanne/Bleich, Sabrina/Sievers, Christoph/Marschall, Ursula/Weidenhammer,Jörg/Fegert,Jörg M. (2012a): Deutsche Traumafolgekostenstudie. Kein Kind mehr - kein(e) Trauma(kosten) mehr? Kiel: Schmidt \& Klaunig.

Habetha, Susanne/Bleich, Sabrina/Weidenhammer, Jörg/Fegert, Jörg M. (2012b): A prevalence-based approach to societal costs occurring in consequence of child abuse and neglect, in: Child and Adolescent Psychiatry and Mental Health 6(1), 35 .

Heim, Christine/Nemeroff, Charles B. (2001): The role of childhood trauma in the neurobiology of mood and anxiety disorders: preclinical and clinical studies, in: Biological Psychiatry 49(12), 1023-1039.

Heim, Christine M./Mayberg, Helen S./Mletzko, Tanja/Nemeroff, Charles B./Pruessner, Jens $C$. (2013): Decreased cortical representation of genital somatosensory field after childhood sexual abuse, in: The American Journal of Psychiatry 170(6), 616-623.

Jud, Andreas/Rassenhofer, Miriam/Witt, Andreas/Münzer, Annika/Fegert, Jörg M. (2016): Häufigkeitsangaben zum sexuellen Missbrauch. Internationale Einordnung, 
Bewertung der Kenntnislage in Deutschland, Beschreibung des Entwicklungsbedarfs, einzusehen unter https://beauftragter-missbrauch.de/fileadmin/Content/ pdf/Pressemitteilungen/Expertise_H\%C3\%A4ufigkeitsangaben.pdf, zuletzt aufgerufen am 9.4.2021.

Jud, Andreas/Fegert, Jörg M./Finkelhor, David (2016): On the incidence and prevalence of child maltreatment: a research agenda, in: Child and Adolescent Psychiatry and Mental Health 10(17).

Katsch, Matthias (2020): Damit es aufhört:Vom befreienden Kampf der Opfer sexueller Gewalt in der Kirche. Berlin: Nicolai.

Kavemann, Barbara/Lohstöter, Ingrid (1984): Väter als Täter. Sexuelle Gewalt gegen Mädchen. Reinbek bei Hamburg: Rowohlt.

Kavemann, Barbara/Graf-van Kesteren, Annemarie/Rothkegel, Sibylle/Nagel, Bianca (2016): Erinnern, Schweigen und Sprechen nach sexueller Gewalt in der Kindheit. Ergebnisse einer Interviewstudie mit Frauen und Männern, die als Kind sexuelle Gewalt erlebt haben. Wiesbaden: Springer VS.

Kempe, C. Henry/Silverman, Frederic N./Steele, Brandt F./Droegemueller, William/Silver, Henry K. (1962): The Battered-Child Syndrome, in: JAMA 181(1), 17-24.

Lempp, Reinhart (1983): Gerichtliche Kinder- und Jugendpsychiatrie. Bern: Huber.

Niehaus, Susanna/Volbert, Renate/Fegert, Jörg M. (2017): Entwicklungsgerechte Befragung von Kindern in Strafverfahren. Berlin: Springer.

Pfeiffer, Elisa/Sachser, Cedric/Rohlmann, Friederike/Goldbeck, Lutz (2018): Effectiveness of a trauma-focused group intervention for young refugees: a randomized controlled trial, in: The Journal of Child Psychology and Psychiatry 59(11), 1171-1179.

Rassenhofer, Miriam/Spröber, Nina/Schneider, Thekla/Fegert, Jörg M. (2013): Listening to victims: Use of a Critical Incident Reporting System to enable adult victims of childhood sexual abuse to participate in a political reappraisal process in Germany, in: Child Abuse \& Neglect 37(9), 654-663.

Rassenhofer, Miriam/Zimmer, Andreas/Spröber, Nina/Fegert,JörgM. (2015): Child sexual abuse in the Roman Catholic Church in Germany: Comparison of victim-impact data collected through church-sponsored and government-sponsored programs, in: Child Abuse \& Neglect 40, 6o-67.

Remschmidt, Helmut/Schmidt, Martin H./Poustka, Fritz (2001): Multiaxiales Klassifikationsschema für psychiatrische Erkrankungen im Kindes- und Jugendalter nach ICD-10 DER WHO mit einem synoptischen Vergleich von ICD-10 und DSM-IV. 4. und 5. Aufl. Bern: Hans Huber.

Stoltenborgh, Marije/Bakermans-Kranenburg, Marian J./van IJzendoorn, Marinus $H$. (2013): The neglect of child neglect: a meta-analytic review of the prevalence of neglect, in: Social Psychiatry and Psychiatric Epidemiology 48(3), 345-355.

Trube-Becker, Elisabeth (1982): Gewalt gegen das Kind:Vernachlässigung, Misshandlung, sexueller Missbrauch und Tötung von Kindern. Heidelberg: Kriminalistik-Verlag. 
Witt, Andreas/Schmid, Marc/Fegert, Jörg M./Plener, Paul L./Goldbeck, Lutz (2014): Temperament und Charaktereigenschaften als protektive Faktoren bei Jugendlichen in stationären Jugendhilfeeinrichtungen, in: Praxis der Kinderpsychologie und Kinderpsychiatrie 63(2), 114-129.

Witt, Andreas/Rassenhofer, Miriam/Allroggen, Marc/Brähler, Elmar/Plener, Paul L./ Fegert,Jörg M. (2019a): The prevalence of sexual abuse in institutions: results from a representative Population-based sample in Germany, in: Sexual Abuse 31(6), 643-661.

Witt, Andreas/Sachser, Cedric/Plener, Paul L./Brähler, Elmar/Fegert,Jörg M. (2019b): Prävalenz und Folgen belastender Kindheitserlebnisse in der deutschen Bevölkerung, in: Deutsches Ärzteblatt 116(38), 635-642.

Witt, Andreas/Jud, Andreas/Finkelhor, David/Brähler, Elmar/Fegert, Jörg M. (2020), Monitoring recent trends: The prevalence of disclosure of sexual abuse in a representative sample of the German population based on indicator 16.2.3 of the UN Sustainable Development Goals (SDG), in: Child Abuse \& Neglect 107, 104575.

Zimmer, Andreas/Lappehsen-Lengler, Dorothee/Weber, Maria/Götzinger, Kai (2014): Sexueller Kindesmissbrauch in kirchlichen Institutionen - Zeugnisse, Hinweise, Prävention. Ergebnisse der Auswertung der Hotline der Deutschen Bischofskonferenz für Opfer sexueller Gewalt. Weinheim/Basel: Beltz Juventa. 